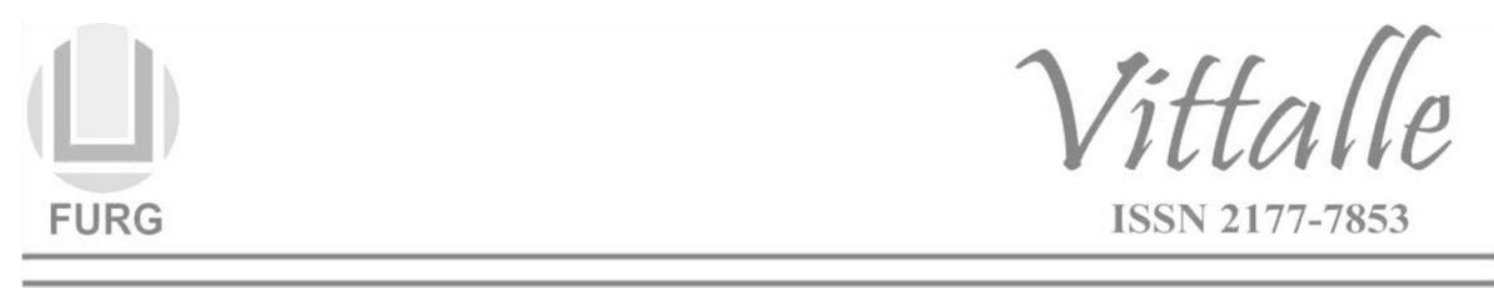

\title{
Adesão ao tratamento no cuidado de pessoas com HIV/AIDS: um estudo em hospital de ensino
}

\author{
Carlos Thommen Corrêa Jankovitz ${ }^{\mathrm{a}^{*}}$, Juliana dos Santos Costa ${ }^{\mathrm{a}}$, Lilian Koifman ${ }^{\mathrm{a}}$, \\ Tania Ventura ${ }^{\mathrm{b}}$
}

${ }^{\text {a}}$ Universidade Federal Fluminense (UFF), Niterói, RJ, Brasil

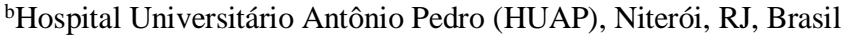

\section{Histórico do Artigo \\ Recebido em: \\ 01/12/2019 \\ Aceito em:}

$24 / 04 / 2020$

Palavras-chave:

Adesão ao tratamento; HIV; AIDS

Keywords:

Treatment adherence; HIV; AIDS

\begin{abstract}
RESUMO
A adesão à terapia antirretroviral (TARV) tem sido um desafio constante à rede de cuidado dos pacientes HIV+. Sabe-se que a posologia inadequada da medicação acarreta não só prejuízos ao indivíduo, como também ao sistema público de saúde. Visto isso, o estudo buscou avaliar a adesão à TARV dos usuários atendidos no serviço especializado de um hospital universitário, analisando os fatores psicossociais que influenciam nesse processo. Foram coletados dados sociodemográficos de 59 pacientes, além dos índices laboratoriais de carga viral e contagem de células TCD4+, entre os anos de 2014 e 2015. Para avaliar correlação estatística entre as variáveis do estudo e a adesão, usaram-se os testes de Mann Whitney, Qui-Quadrado de Person e Odds Ratio. Observou-se que 50,85\% dos entrevistados não aderiam à terapia, sendo as dificuldades com a TARV e a mudança de rotina pela medicação, fatores associados à não-adesão $(\mathrm{p}<0,05)$. Variáveis como idade, mudança de profissional e compreensão das taxas laboratoriais não se mostraram significantes $(\mathrm{p}>0,05)$. Nesse contexto, torna-se evidente a importância de se conhecer os pontos de instabilidade dos serviços de HIV/AIDS, visando, assim, aprimorar as condições da assistência ofertadas por estes.
\end{abstract}

Adherence to treatment in the care of people with HIV/AIDS: a study in a teaching hospital

\section{ABSTRACT}

Adherence to antiretroviral therapy has been a constant challenge to the care network of HIV+ patients. It is known that the inadequate dosage of the medication causes harm not only to the individual, but also to the public health system. In this sense, the study sought to assess adherence to ART from users at the specialized service of a university hospital, analyzing the psychosocial factors that influence this process. Sociodemographic data, laboratory viral load index and TCD4+ cell count were collected from 59 patients between the years 2014 and 2015. To assess statistical correlation between the study variables and adherence, the Mann Whitney, Person's Chi-Square and Odds Ratio tests were used. It was observed that $50.85 \%$ of the interviewees did not adhere to therapy, with difficulties in ART regimes and the change of routine due to medication, factors associated with non-adherence $(\mathrm{p}<0.05)$. Variables such as age, change of professional and understanding of rates laboratory tests were not significant $(p>0.05)$. In this context, the importance of knowing the points of instability of HIV/AIDS services becomes evident, in order to improve the conditions of assistance offered by these.

\section{Introdução}

A infecção pelo vírus da imunodeficiência humana (Human Immunodeficiency Virus - HIV) ainda é uma preocupação humanitária global, fato justificado pelos 1,7 milhões de novos casos a cada ano (1). Esse número anual de infecções já foi imensamente maior, com pico de 2,9 milhões de casos apenas em 1997 (1). No Brasil, desde a primeira ocorrência de AIDS notificada em 1980 até junho de 2019, já foram identificados 966.058 casos de HIV (2), com uma média anual de 39 mil novos casos de

\footnotetext{
* Autor correspondente: carlosthommen@ hotmail.com (Jankovitz C.T.C.)
} 
AIDS nos últimos cinco anos. Entre 2007 e junho de 2019, 300.496 casos da infecção foram notificados, e destes, 136.902 (45,6\%) ocorreram somente na região Sudeste do país (2). Diante dessa realidade, os medicamentos responsáveis por reduzir a replicação viral e, assim, interferir no curso natural da doença tornaram-se imprescindíveis no combate à pandemia do HIV. Principalmente a partir surgimento da terapia combinada em 1996, a evolução dos esquemas antirretrovirais permitiu não só reduzir os altos índices de mortalidade e morbidade dos pacientes infectados pelo vírus, como também promoveu a queda de novos casos ao longo dos anos (3). Diante desse cenário, torna-se fundamental salientar a importância da Terapia Antirretroviral (TARV) frente à epidemia do HIV, essencialmente quando se analisa a estratégia de tratamento e prevenção adotada pelo governo brasileiro.

O Brasil oferta universal e gratuitamente, por meio da Lei $\mathrm{n}^{\circ} 9.313$ de 1996, os medicamentos necessários ao tratamento dos portadores do HIV e doentes de AIDS. Essa política aplicada pelo país gerou grandes resultados frente às comorbidades e expectativa de vida dos pacientes, expressos pela diminuição da morbimortalidade, das internações hospitalares e da transmissão vertical do vírus, além da redução do risco da transmissão horizontal e do desenvolvimento de HIV resistente aos medicamentos antirretrovirais $(4,5)$. Dessa forma, com o consequente aumento da longevidade dos indivíduos em razão da TARV, a infecção pelo HIV começou a ser manejada como uma doença crônica, que demanda, entre outros cuidados, acompanhamento médico regular e mudanças significativas no estilo de vida do paciente.

Tendo isso em vista, a adesão à TARV tornou-se uma preocupação constante dos profissionais de saúde para com os pacientes soropositivos. Segundo a Organização Mundial da Saúde (OMS), os tratamentos a longo prazo apresentam grande falha de adesão na população em geral, resultando em taxas de 50,0\% à não-adesão (6). Muitos autores entendem a não-adesão ao tratamento de doenças crônicas, incluindo o contexto de HIV/AIDS contemporâneo, como um problema sério de saúde pública, não só por aumentar os custos com os cuidados do paciente, mas também por elevar as complicações acarretadas pela doença, reduzindo drasticamente a qualidade de vida desses indivíduos $(7,8)$. Sendo assim, a não-adesão à TARV é considerada uma falha desastrosa na garantia das boas condições de vida da sociedade como um todo, mas principalmente, dos portadores do vírus. Portanto, compete às equipes de saúde, ao Estado e aos indivíduos soropositivos alcançar efetiva adesão à TARV, compreendida por tomar corretamente os medicamentos antirretrovirais prescritos, seguir as doses corretas pelo tempo preestabelecido, bem como aderir ao serviço de saúde especializado (3).

Diante de tamanha responsabilidade, o Ministério da Saúde criou, em 1994, os Serviços de Assistência Especializada (SAE) em HIV/AIDS. O SAE é uma unidade assistencial de caráter ambulatorial que tem como objetivo, por meio da atuação de uma equipe multiprofissional, realizar ações de assistência, prevenção e tratamento às pessoas com HIV/AIDS (9). Visto isso, é notório a importância desses serviços frente ao tratamento dos portadores de HIV, funcionando tanto como espaços estratégicos de informação e execução de intervenções no campo da adesão, discutindo, principalmente, sobre importância da adesão e a adequação do tratamento à rotina de vida do paciente, quanto como locais de atendimento ambulatorial clínico a essas pessoas (10).

Nesse contexto, o objetivo do presente estudo foi avaliar a adesão à TARV dos pacientes portadores de HIV, analisando os fatores psicossociais que influenciam nesse processo complexo, em um Serviço de Assistência Especializado (SAE) em HIV/AIDS de um hospital universitário, e, com isso, proporcionar insumos práticos que viabilizem o aprimoramento das condições de assistência ofertadas por este. 


\section{Materiais e métodos}

\section{Estratégias}

Trata-se de um estudo transversal, exploratório, com abordagem quantitativa, desenvolvido pelo Serviço Ambulatorial Especializado (SAE) do Hospital Universitário Antônio Pedro (HUAP), na cidade de Niterói-RJ, com usuários que obedeciam aos critérios da pesquisa. Os participantes foram recrutados a partir de amostragem por conveniência, à medida que compareciam ao serviço para atendimento, totalizando 74 entrevistados. As recusas (7) foram respeitadas. O período da coleta de dados foi compreendido entre 2014 e 2015, sendo esses coletados no dia das consultas, anteriormente ao horário agendado e em uma sala que garantia a privacidade dos pacientes.

\section{Critérios de Inclusão e Exclusão}

Foram incluídos no estudo indivíduos maiores de 18 anos, sabidamente soropositivos ao HIV e em uso de TARV há pelo menos 6 meses, de ambos os sexos e que estivessem fazendo acompanhamento na Coordenação de AIDS do HUAP.

Como critérios de exclusão, removeram-se aqueles que faleceram, não cumpriam tratamento no HUAP, não realizaram os exames laboratoriais no período de 2014/2015 ou detinham dados laboratoriais incompletos (Figura 1).

Sendo assim, daqueles 74 pacientes inicialmente entrevistados, 59 foram considerados para as análises do estudo.

\section{Coleta de Dados e Questionário}

Os dados foram coletados por meio de entrevista composta por um questionário inspirado no teste de Morisky-Green (11), desenvolvido e executado pela equipe multiprofissional da Coordenação de AIDS/HUAP e alunos de medicina da Universidade Federal Fluminense (UFF). Avaliaram-se características como gênero, idade, letramento, compreensão das taxas laboratoriais, assiduidade no uso dos medicamentos, entre outros pontos que poderiam auxiliar ou prejudicar a adesão ao tratamento, que, por fim, tornaram-se as variáveis independentes deste estudo.

Adicionalmente, foi feito registro e análise dos dados laboratoriais coletados dos prontuários no período estabelecido e elaborado um banco em planilha do Excel for Windows. 


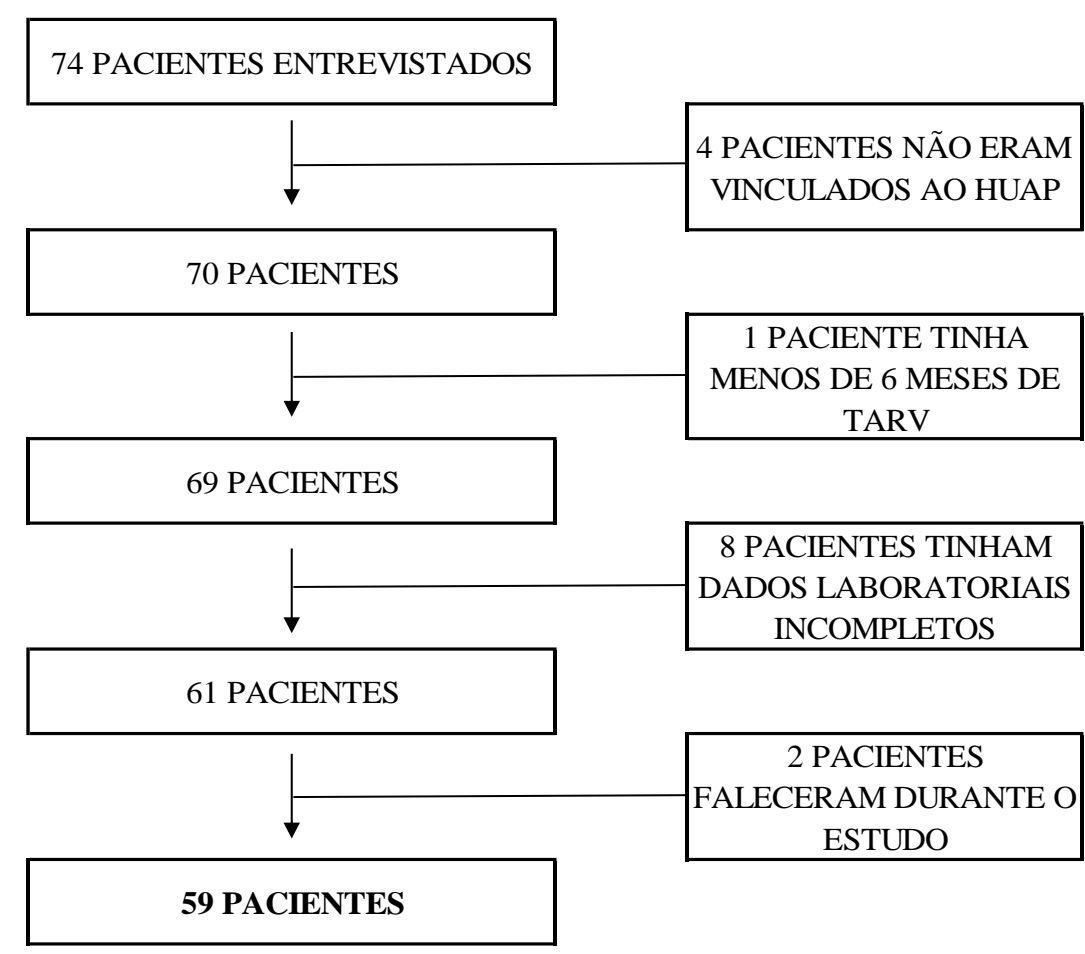

Figura 1 - Fluxograma da seleção por exclusão dos participantes

\section{Avaliação da Adesão}

Como não há uma metodologia considerada padrão ouro nos serviços de saúde do SUS (8), o acompanhamento e a avaliação da adesão ao tratamento tornaram-se grandes desafios para quem trabalha em saúde, uma vez que os métodos ou procedimentos disponíveis geram muitas vezes resultados parciais e apresentam discordância entre suas vantagens e desvantagens a cada serviço. Desta forma, Coutinho et al. (12) sugere que se utilize a carga viral como método de análise da adesão, sendo este um dos mais confiáveis. Com isso, optou-se por mensurar a adesão, considerada a variável dependente do estudo, a partir de valores de carga viral ( $<40$ cópias $/ \mathrm{ml})$ e taxa de linfócitos TCD4+ (> 350 células/ $\mu 1)$ dos pacientes.

\section{Análises Estatísticas}

Os dados dos pacientes foram exportados do Excel for Windows para o software Package for Social Science (SPSS), versão 20.0. As associações foram consideradas estatisticamente significantes quando o valor de $\mathrm{p}<0,05$. Para as variáveis numéricas, foi realizado o teste não paramétrico de Mann Whitney, que compara das médias entre os pacientes que não tiveram adesão e os que aderiram. Para as variáveis categóricas, conferiu-se associação através do teste Qui-Quadrado de Person, além de ser realizado o Odds Ratio para mensuração da medida de efeito dessas variáveis. O intervalo de confiança de $95 \%$ foi estimado pelo método Bootstrap.

\section{Aspectos Éticos}

A todos os participantes da pesquisa foi garantido o caráter sigiloso dos dados e o anonimato. O projeto foi submetido ao Comitê de Ética do HUAP/UFF, com CAAE: 04841712.2.0000.5243. 
Vittalle - Revista de Ciências da Saúde v. 32, n. 2 (2020) 27-35

\section{Resultados e discussão}

Este estudo teve o intuito de avaliar a adesão à TARV dos usuários atendidos pelo SAE - HIV/AIDS, localizado na Coordenação de AIDS do Hospital Universitário Antônio Pedro (CAIDS/HAUP) em Niterói, e, com isso, compreender o processo de tratamento do HIV por diversas vertentes. Tendo isso vista, vale salientar que alguns dados individuais não foram levados em consideração, como a orientação sexual e a escolaridade dos participantes, visto que não se demonstraram relevantes frente à pesquisa em questão. Reconhece-se que essa escolha implica em algumas limitações epidemiológicas do estudo, sem excluir, contudo, sua importância essencial: avaliar a adesão dos pacientes frente à terapia antirretroviral em um hospital universitário. Portanto, faz-se necessário uma análise mais criteriosa dos diversos pontos abordados, levando em consideração o cenário de pesquisa exposto.

De acordo com as variáveis sociodemográficas do estudo, 29 indivíduos $(49,15 \%)$ pertenciam ao sexo masculino e $30(50,85 \%)$ ao sexo feminino. No Brasil, há um predomínio de homens quando se analisa o perfil de pessoas portadoras de HIV (13), o que contrapõe o achado desse estudo, cuja maioria de soropositivos é do sexo feminino. Em relação à faixa etária, dentre os grupos de gênero, a idade média masculina era de 49,32 anos, variando entre 20 e 74 anos (desvio padrão de 13,92). Já a idade média feminina ficou em torno de 40,36 anos, com números entre 18 e 71 anos (desvio padrão de 15,79). Além disso, dentre os entrevistados que frequentavam a CAIDS/HAUP, mais de três quartos $(76,27 \%)$ confirmaram usufruir de algum benefício oferecido pelo governo, sendo o vale-transporte o mais relatado. Diversos autores apontam que fatores como o suporte social aos soropositivos reforçam a manutenção e o sucesso no acompanhamento clínico dos pacientes durante o tratamento (3), o que estrutura a importância dessas políticas públicas como ferramentas indispensáveis para o cuidado da população portadora do vírus. Desse modo, ficam claros o papel e a enorme responsabilidade do Estado para com os indivíduos HIV positivo, uma vez que medidas públicas que auxiliem o complexo processo de tratamento ao HIV podem influenciar, demasiadamente, no curso da terapia antirretroviral desses pacientes.

Em relação às variáveis clínicas da pesquisa, 62,71\% dos pacientes mantiveram sua carga viral $<40$ cópias $/ \mathrm{ml}$ e 50,85\% apresentaram contagem de células TCD4+> 350/ $\mu 1$ durante o período analisado. Esses dados foram fundamentais para avaliar a adesão à terapia antirretroviral na amostra coletada, sendo esta observada em 49,15\% dos entrevistados (Tabela 1). Em uma pesquisa avaliativa desenvolvida pela Universidade de São Paulo (USP), que buscou medir a adesão ao tratamento antirretroviral em ambulatórios do sistema público de saúde brasileiro por meio da análise da administração de comprimidos de 1.972 pacientes provenientes de 322 serviços localizados em 7 estados brasileiros, a taxa de aderência ao tratamento encontrada ficou em torno de $73 \%$ (14). Esse resultado é cerca de 50\% superior ao constatado no grupo amostral presente. Em contra partida, o estudo evidenciou, também, que em serviços pequenos, como o SAE/CAIDS/HUAP, com menos de 100 pacientes portadores do vírus, existe um maior risco de não-adesão à TARV. Mediante isso, com o intuito de avaliar os fatores relacionados a inferior adesão desse serviço, realizaram-se diversas associações estatísticas entre a adesão dos pacientes e as variáveis sociodemográficas declaradas por estes. Como resultado, a adesão à terapia antirretroviral mostrou-se positivamente correlacionada ao sexo masculino $(\mathrm{p}=0,026)$, conforme evidenciado na Tabela 2. Sendo assim, percebeu-se que haveria maior probabilidade de constatar adesão ao tratamento se o paciente fosse homem, evento que contrapõe diversos estudos 
na literatura $(15,16)$. Já estudos que apresentaram associação similar a esse, o fato de que as mulheres necessitam, na maioria das vezes, administrar as rotinas familiares, negligenciando o autocuidado, tornou-se o argumento mais aceitável para explicar tal diferença (5). Em relação à idade dos pacientes, não houve associação que apresentasse significância estatística à adesão.

Tabela 1 - Categorização clínica de pacientes com HIV.

\begin{tabular}{|c|c|c|}
\hline Variável & $\mathbf{n}$ & $\%$ \\
\hline \multicolumn{3}{|l|}{ Contagem de TCD4+ (células/ $\mu \mathrm{l})$} \\
\hline$>350$ & 30 & $50,85 \%$ \\
\hline$\leq 350$ & 29 & $49,15 \%$ \\
\hline \multicolumn{3}{|l|}{ Carga Viral do HIV (cópias/ml) } \\
\hline$<40$ & 37 & $62,71 \%$ \\
\hline$\geq 40$ & 22 & $37,29 \%$ \\
\hline \multicolumn{3}{|l|}{ Adesão ao tratamento } \\
\hline Sim & 29 & $49,15 \%$ \\
\hline Não & 30 & $50,85 \%$ \\
\hline Total de pacientes analisados & 59 & $100,00 \%$ \\
\hline
\end{tabular}

Tabela 2 - Associação entre variáveis sociodemográficas com adesão à terapia antirretroviral.

\begin{tabular}{|c|c|c|c|}
\hline Variável & Odds Ratio & IC (95\%) & p-valor* \\
\hline \multicolumn{4}{|c|}{ Sexo/Gênero dos participantes } \\
\hline Masculino & 3,28 & \multirow{2}{*}{$1,13-9,57$} & \multirow{2}{*}{0,026} \\
\hline Feminino & 1 & & \\
\hline \multirow{2}{*}{\multicolumn{4}{|c|}{$\begin{array}{l}\text { Dificuldades para seguir com o } \\
\text { tratamento }\end{array}$}} \\
\hline & & & \\
\hline Sim & 1 & \multirow{2}{*}{$1,69-43,17$} & \multirow{2}{*}{0,003} \\
\hline Não & 8,54 & & \\
\hline \multicolumn{4}{|c|}{ Dificuldades com a medicação } \\
\hline Sim & 1 & \multirow{2}{*}{$1,56-25,41$} & \multirow{2}{*}{0,005} \\
\hline Não & 6,41 & & \\
\hline \multicolumn{4}{|c|}{$\begin{array}{l}\text { Trocou de médico durante o } \\
\text { tratamento para HIV/AIDS }\end{array}$} \\
\hline Sim & 1 & \multirow{2}{*}{$0,76-6,43$} & \multirow{2}{*}{0,137} \\
\hline Não & 2,22 & & \\
\hline \multicolumn{4}{|c|}{$\begin{array}{l}\text { Fala sobre a sua soropositividade a } \\
\text { outros médicos }\end{array}$} \\
\hline Sim & 1,8 & \multirow{2}{*}{$0,54-5,92$} & \multirow{2}{*}{0,329} \\
\hline Não & 1 & & \\
\hline \multicolumn{4}{|c|}{$\begin{array}{l}\text { Sabe o significado das taxas de CD4, } \\
\text { CD8 e carga viral }(\mathrm{CV})\end{array}$} \\
\hline Sim & 1,41 & \multirow{2}{*}{$0,50-3,96$} & \multirow{2}{*}{0,506} \\
\hline Não & 1 & & \\
\hline
\end{tabular}

*Teste Qui-quadrado de Pearson 
Conforme o último manual de adesão ao tratamento para pessoas vivendo com HIV e AIDS publicado pelo Ministério da Saúde (8), a não-adesão pode estar atrelada a diversos fatores, sendo eles socioeconômicos e culturais, psicológicos, institucionais ou, também, advindos da relação entre o profissional de saúde e o usuário. Com isso, compreender os fatores que influenciam a não-adesão torna-se fundamental no direcionamento da força de trabalho dos profissionais da saúde e das políticas públicas necessárias para lidar com a situação de forma eficiente e resolutiva. Paralelamente, ao analisar questões referentes à terapia antirretroviral, estudos similares a esse $(17,18)$ indicaram falhas de adesão quando se correlaciona fatores associados ao não cumprimento dos horários de administração do medicamento e à baixa consciência da importância do tratamento. Frente a isso, torna-se justificável que apenas 49,15\% dos entrevistados da pesquisa apresentam efetiva adesão ao tratamento, uma vez que as dificuldades com a TARV e a mudança de rotina pela medicação se mostram intimamente atrelados ao baixo nível de adesão dos pacientes $(\mathrm{p}<0,05)$. Portanto, os serviços de saúde se tornam essenciais nesse contexto, já que são responsáveis por promover participação ativa do usuário no tratamento, além de desenvolver a capacitação necessária desse para lidar com um regime terapêutico complexo e de difícil adaptação (7).

Nesse viés, convém indicar a literacia funcional em saúde, descrita como a capacidade dos indivíduos em obter, processar e compreender informações e serviços necessários para a tomada de decisões apropriadas ao autocuidado, como um fator de imensa interferência quando se estuda a adesão ao tratamento do HIV (8). No estudo presente, notou-se que mais da metade $(50,85 \%)$ dos participantes não conheciam o nome dos medicamentos que utilizavam, mesmo sendo tratados há mais de 6 meses com estes, e $76,27 \%$ não conferiam a receita médica para a confirmação de dosagem, horário ou nomenclatura dos fármacos. Sabe-se que há uma tendência erronia na prática posológica de medicamentos quando as pessoas não compreendem a adequada maneira de realizála (5). Desse modo, fornecer informações básicas sobre a TARV mostra-se uma atividade fundamental no combate à não-adesão. Nessa pesquisa, a dificuldade com a posologia da medicação antirretroviral evidenciou associação significativa com a nãoadesão à terapia (p-valor=0,005). Adicionalmente, sabe-se que essa dificuldade encontrada abrange, entre diversas questões, pontos relacionados à pessoa portadora do vírus; à doença, se presente; e ao tratamento, incluindo, também, o serviço e os profissionais de saúde como fatores influenciadores (19).

Dessa forma, além de informar, os profissionais em saúde, atuando como equipe multiprofissional, devem estabelecer vínculo com o paciente, permitindo que este se empodere de senso crítico e responsabilidade para com sua saúde (4). Visando alcançar tal objetivo, cabe considerar alguns aspectos que, possivelmente, interfiram de forma negativa na criação e manutenção dessa relação. A mudança de profissional durante o acompanhamento médico e a insegurança do usuário quando há necessidade de se expor sobre a soropositividade são variáveis que necessitam atenção do serviço, visto que podem, muitas vezes, desencadear falhas de relação e vínculo entre o paciente e o profissional. Dentre os entrevistados do estudo, 38,98\% afirmaram já ter trocado de médico durante o tratamento e $23,72 \%$ não relatam a outros médicos sobre sua condição de soropositivo, o que pode ser abarcado quando se observa que 16,95\% diz já ter sofrido algum preconceito por parte do profissional. Desse modo, fica nítido o papel de respeito e empatia da equipe de saúde como sendo corresponsável na estruturação da maneira de como o usuário lida com o HIV e, consequentemente, adere à terapia antirretroviral.

Dito isso, é notório que as estratégias terapêuticas empregadas pelos serviços de saúde 
especializados em HIV/AIDS devem ser muito bem estruturadas e esquematizadas, considerando significante a realidade do paciente, o serviço em que ele é atendido e o suporte social disponível a este. Isso implica, portanto, na formulação de um plano individualizado de execução compartilhada e colaborativa, visando fortalecer $\mathrm{o}$ autocuidado e autonomia do usuário (3).

\section{Considerações finais}

O estudo indicou baixa adesão à TARV dos pacientes que frequentam o Serviço de Assistência Especializada da Coordenação de AIDS do Hospital Universitário Antônio Pedro quando comparada à taxa de aderência nacional. As dificuldades com a manutenção da terapia antirretroviral e com a rotina da medicação foram referidas como os principais fatores associados ao resultado do estudo. Portanto, fica estruturado que a adesão, sendo ainda um enorme desafio da rede de cuidado, reflete as condições sociodemográficas individuais dos usuários, constituindo um processo dinâmico que necessita de auxílio por parte de equipe multiprofissional, apoio governamental e quaisquer outros suportes sociais disponíveis ao paciente portador do HIV.

Nesse contexto, torna-se evidente a importância de se conhecer os pontos de instabilidade de cada ambulatório especializado em HIV/AIDS da rede pública de saúde e, a partir deles, promover intervenções de aprimoramento à adesão ao tratamento e às condições da assistência ofertadas por esses serviços. Nesse sentido, a CAIDS/HUAP detém elevada responsabilidade para com os pacientes, necessitando ampliar a atuação e intensificar sua influência no acompanhamento individualizado dos seus usuários. Deve-se, portanto, remanejar as questões deficitárias apontadas no serviço, visando, assim, garantir a todos o tratamento adequado e contribuir na promoção de qualidade de vida em todo o seu aspecto biopsicossocial.

\section{Agradecimentos}

Ao PIBIC - Universidade Federal Fluminense pela bolsa concedida; ao Instituto de Saúde Coletiva - UFF pelo incentivo e aporte técnico; aos pacientes da CAIDS/HUAP que contribuíram com conhecimento singular, que extrapolava o domínio teórico, mas fomentaram nosso enriquecimento como seres humanos; e a todos aqueles que contribuíram direta ou indiretamente com a elaboração desse trabalho, que muito irá ofertar na construção no cuidado.

\section{Conflitos de interesse}

A equipe da pesquisa não tem qualquer conflito de interesse.

\section{Referências}

1. UNAIDS. Joint United Nations Programme on HIV/AIDS [Internet]. 2019 [Acessado em 02 de abril de 2020]. Disponível em:< https://www.unaids.org/sites/default/files/media_asset/2019-UNAIDSdata_en.pdf $>$.

2. Ministério da Saúde, Secretaria de Vigilância em Saúde. Boletim Epidemiológico HIV. Brasília: Ministério da Saúde, 2019.

3. Camargo LA, Capitao CG, Filipe EMV. Saúde mental, suporte familiar e adesão ao tratamento: associações no contexto HIV/Aids. Psico-USF 2014; 19(2): 221-232.

4. Cancian NR, Beck ST, Santos GS, Bandeira D. Importância da atenção multidisciplinar para resgatar o paciente com hiv/aids apresentando baixa adesão à terapia antirretroviral. Rev. de Atenção à Saúde 2015; 13(45): 55-60. 
5. Romeu GA, Tavares MM, Carmo CP, Magalhães KDN, Nobre ACL, Matos VCD. Assessment of adherence to antiretroviral therapy for patients with HIV. Rev Bras Farm Hosp Serv Saude 2019; $3(1)$.

6. Tavares NUL, Bertoldi AD, Mengue SS, Arrais PSD, Luiza VL, Oliveira MA, et al. Fatores associados à baixa adesão ao tratamento farmacológico de doenças crônicas no Brasil. Rev Saude Pública 2016; 50(supl 2): 10.

7. Liberato SMD, Souza AJG, Gomes ATL, Medeiros LP, Costa IKF, Torres GV. Relação entre adesão ao tratamento e qualidade de vida: revisão integrativa da literatura. Rev. Eletr. Enf 2014; 16(1): 191-8.

8. Ministério da Saúde. Secretaria de Ciência, Tecnologia e Insumos Estratégicos. Departamento de Ciência e Tecnologia. Síntese de evidências para políticas de saúde: adesão ao tratamento medicamentoso por pacientes portadores de doenças crônicas. Brasília: Ministério da Saúde, 2016.

9. Abrão FMS, Angelim RCM, Cardoso MD, Queiroz SBA, Freitas RMM, Oliveira DC. Características estruturais e organizacionais de serviços de assistência especializada em HIV/AIDS na cidade de Recife, Brasil. Rev Baiana Saúde Pública 2014; 38: 140-154.

10. Bonolo PF, Gomes RRFM, Guimarães MDC. Adesão à terapia anti-retroviral (HIV/aids): fatores associados e medidas da adesão. Epidemiol. Serv. Saúde 2007; 16(4): 267-278.

11. Morisky DE, Ang A, Krousel-Wood M, Ward HJ. Predictive validity of a medication a d herence measure in anout patient setting. J ClinHypertens (Greenwich) 2008; 10 (5): 348-54.

12. Coutinho MFC, O'Dwyer G, Frossard V. Tratamento antirretroviral: adesão e a influência da depressão em usuários com HIV/Aids atendidos na atenção primária. Saúde debate 2018; 42(116): 148-161.

13. Menezes EG, Santos SR, Melo GZ, Torrente G, Pinto AS, Goiabeira YN. Fatores associados à não adesão dos antirretrovirais em portadores de HIV/AIDS. Acta Paul Enferm 2018; 31(3): 299-304.

14. Nemes MIB, Carvalho HB, Souza MFMS. Anti-retroviral therapy adherence in Brazil. AIDS 2004; 18(supl 3):15-20.

15. Da Silva JVF, Júnior FJMN, Rodrigues APRA. Fatores de não adesão ao tratamento antirretroviral: desafio de saúde pública. Ciências Biológicas e da Saúde 2014; 2(1): 165-17.

16. Colombrini MRC, Lopes MHBM, Figueiredo RM. Adesão à terapia antiretroviral para HIV / AIDS. Rev Esc Enferm USP 2006; 40 (4): 576-81.

17. Remor KVT, Ogliari LC, Sakae TM, Galato D. Adesão aos antirretrovirais em pessoas com HIV na grande Florianópolis. Arquivos Catarinenses de Medicina 2017; 46(2): 53-64.

18. Moraes DCA, Oliveira RC, Da Motta MCS, Ferreira OLC, Andrade MS. Terapia antirretroviral: a associação entre o conhecimento e a adesão. Revista de Pesquisa Cuidado é Fundamental Online 2015; 7(4): 3563-3573.

19. Paschoal EP, Santo CCE, Gomes AMT, Santos EI, Oliveira DC, Pontes APM. Adesão à terapia antirretroviral e suas representações para pessoas vivendo com HIV/AIDS. Esc Anna Nery 2014; 18(1): 32-40. 